\title{
Editorial: Journal of Classification Vol. 38-1
}

\author{
Paul D. McNicholas ${ }^{1}$
}

Accepted: 26 March 2021 / Published online: 9 April 2021

(C) The Classification Society 2021

With a new year comes new hope, especially in this pandemic era. The first issue of 2021 contains a total of ten articles, and the same number is anticipated for the remaining issues this year and quite possibly beyond. The first article in this issue, by Ahn, Park and Hur, introduces a clever algorithm for the notoriously difficult problem of imbalanced data. The second paper, by Aydemir, proposes the polygon area metric as a means of assessing classification performance. In the third paper, Ghassabeh and Rudzicz introduce a subspace constrained mean shift algorithm. The fourth paper, by Ma and Ryoo, introduces an algorithm for the perhaps-understudied problem of spherical classification. In the next paper, Fernández-Durán and Gregorio-Domínguez introduce a clustering algorithm, based on circular statistical models, for consumer segmentation.

The sixth paper, by Subedi and McNicholas, considers a Bayesian alternative to the nearubiquitous expectation-maximization (EM) algorithm (Dempster et al. 1977) for parameter estimation in model-based clustering. After that, Martín-Fernández provides a review of the compositional data analysis book by Greenacre (2018). In the eighth paper, D'Ambrosio, Amodio, Iorio, Pandolfo and Siciliano propose an extension of the adjusted Rand index (ARI; Hubert and Arabie 1985) to fuzzy partitions - remarkably, this is the first of two papers in this issue that extends the ARI. The next paper, by Biernacki, Marbac and Vandewalle, introduces a very nice approach for visualization of model-based clustering results. In the tenth and final paper of this issue, Robert, Vasseur and Brault extend the ARI to co-clustering, i.e., they develop an approach for measuring the agreement between two co-clustering partitions.

\section{References}

Dempster, A.P., Laird, N.M., Rubin, D.B. (1977). Maximum likelihood from incomplete data via the EM algorithm. Journal of the Royal Statistical Society: Series B, 39(1), 1-38.

Greenacre, M. (2018). Compositional Data Analysis in Practice. Boca Raton: Chapman \& Hall/CRC Press. Hubert, L., \& Arabie, P. (1985). Comparing partitions. Journal of Classification, 2(1), 193-218.

Publisher's Note Springer Nature remains neutral with regard to jurisdictional claims in published maps and institutional affiliations.

Paul D. McNicholas

paul@math.mcmaster.ca 